\title{
Demo: Automated Valet Parking and Charging
}

\author{
Stephan Rottmann, Julian Timpner, and Lars Wolf \\ Institute of Operating Systems and Computer Networks \\ Technische Universität Braunschweig \\ Braunschweig, Germany \\ Email: (rottmann|timpner|wolf)@ibr.cs.tu-bs.de
}

\begin{abstract}
A model electric vehicle performing automated valet parking and charging is demonstrated. The vehicle's missions are transmitted using WLAN based vehicle-toinfrastructure communications by a server back-end which has been developed in the EU FP7 project V-Charge.
\end{abstract}

\section{The V-Charge Project}

The European Union FP7 project V-Charge ${ }^{1}$ (Automated Valet Parking and Charging for e-Mobility) offers a sophisticated combination of public transport and individual electrical mobility by introducing automated valet parking based on close-to-market sensors and coordinated charging strategies. This system allows drivers to drop off (and to pick up) their electric vehicle in front of a public transport hub (e.g., an airport) without taking care of parking or recharging. The vehicle executes these tasks autonomously. This implies three major fields of research: (i) vehicle functionality, e.g., on-board localization and planning, (ii) logistics, scheduling of parking and charging resources, and (iii) infrastructure, a secure and reliable framework to store and share a database of parking area information. [1]

In this demo, we present a research and development platform showcasing the abovementioned aspects of the actual V-Charge project while also providing an educational tool for students to learn about automated driving, localization, and vehicular communications. Our platform consists of a vehicle system, that is based on a 6-wheel-drive chassis equipped with a computing and sensor platform, as well as a server system, providing the vehicle with mission goals and a remote monitoring station. Using the server application, a destination such as a parking spot or a charging station can be set, which the vehicle will then automatically navigate and maneuver to. In case of a charging task, the vehicle will also be charged automatically. Compared to the actual V-Charge vehicles, which are based on the VW Golf platform, our miniature vehicles allow cheap, easy and rapid development and prototyping, which is especially useful as an educational tool.

\section{Demonstration Platform}

The vehicle system is based on a Dagu Wild Thumper 6WD Chassis, depicted in Figure 11. The mobile platform is controlled by a Raspberry Pi Single Board Computer (SBC) running Linux (see $C$ in Figure 1). For communication purposes, it is equipped with a USB WLAN dongle. Steering is done by applying different speeds to the wheels on each side of the vehicle, as it does not have a steering shaft. A block diagram of the vehicle is shown in Figure 2. The gray box on

\footnotetext{
${ }^{1}$ http://www.v-charge.eu
}

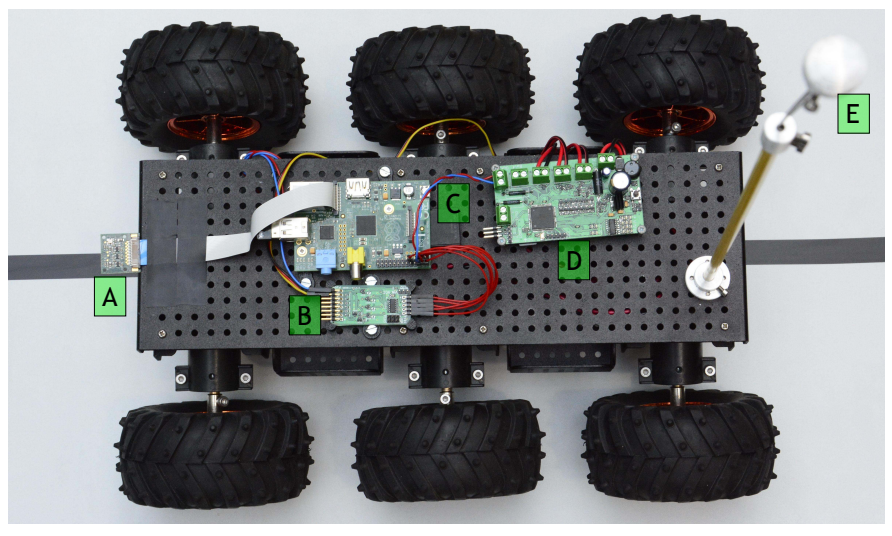

Figure 1. Wild Thumper. A: Camera, B: PWM controller, $C$ : Raspberry Pi SBC, $D$ : Energy Management Module, $E$ : Current collector

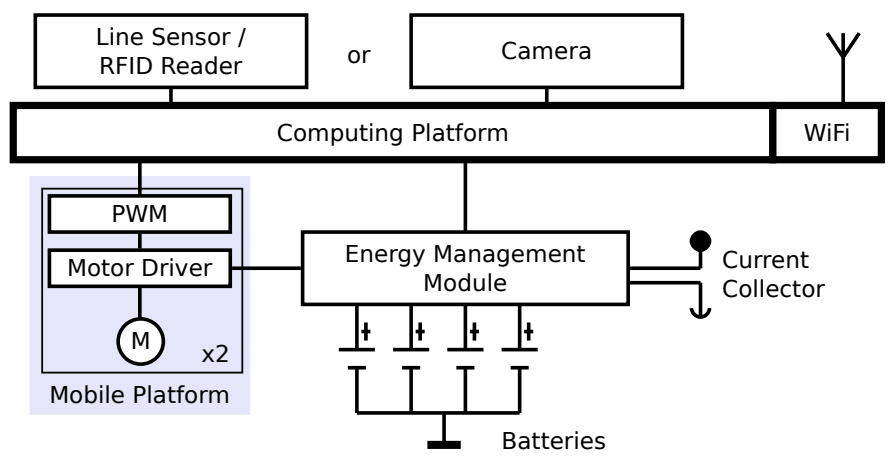

Figure 2. Block diagram of platform components

the left depicts the mechanical platform components including the motors for driving and its control modules.

\section{A. Energy Management Module}

The Energy Management Module (EMM) (D/E) supplies the computing platform and all other components attached to the vehicle with energy. Up to four batteries can be connected to the module of which the most appropriate one is chosen. This is determined by the charging state, usage history (charging cycles) and other factors of each battery. The module supports different kinds of batteries (NiMH, LiPo and SLA). The EMM has two outputs for energy which can be switched on and off. One output has a maximum current of about $2 \mathrm{~A}$, the other one can deliver more than $20 \mathrm{~A}$. The former one is used for powering the controlling SBC, the latter one for driving. All currents and voltages are being logged which allows the system to estimate the remaining runtime 
of the vehicle. To enable automated charging of the vehicle's batteries, bumper cars-like current collectors have been created and mounted on the vehicle. The charging station consists of two pieces of wire mesh above and below the vehicle. The meshes are connected to a voltage source to power the EMM.

\section{B. Trajectory Following}

The vehicle is equipped with a sensor system which is able to detect lines on the ground representing a road network. Tags are used to identify the vehicle's position. A digital version of the map is stored on-board as a directed graph. A path to the given destination is calculated using Dijkstra's algorithm.

We have developed two versions of line sensors. The first one consists of eight reflective optical sensors which are mounted close to the ground. They are attached to an eight bit Microcontroller Unit (MCU) which samples the data of the sensors and transfers the measurements to the SBC. Line segments of the road network are identified via RFID tags. An RFID reader is placed on the same Printed Circuit Board (PCB) as the optical sensors.

The second system for line detection is closer to the real world car since it is using computer vision (see camera $A$ in Figure 11. It is using the open source computer vision libraries OpenCV for recognizing the line depicting the road network and tags for identifying the road segments.

\section{Motor Control}

MOSFET based H-bridges are used to power the motors of the chassis. A Pulsewidth Modulation (PWM) signal for setting the motor speed is generated by an external MCU $(B)$ since the used SBC does not have multiple hardware PWM channels. The external circuit can additionally generate signals for up to three RC servos.

\section{LOGISTICS AND COMMUNICATION}

To enable the automated valet parking as described in Section I. Vehicle-to-Infrastructure (V2I) communications are required to provide the vehicle with mission information and to aggregate vehicle sensor data. The server is responsible for the scheduling of parking and charging resources. All in-vehicle modules of the actual V-Charge vehicles, such as sensors, localization and on-board planning, as well as the serverside components (e.g., path planner, parking management system) share data using the Data Distribution Service (DDS) middleware. On the wireless link between server and vehicles, however, DDS has some drawbacks such as lack of disruptiontolerance and of multi-hop support. Thus, DDS is used both on the vehicle and the server side, while a Disruption-Tolerant Network (DTN) is used for transporting DDS data on the wireless link, as depicted by Figure 3 . In the demonstrator, the DTN transport can be disabled, however, to enable end-toend DDS transmissions over a WLAN link.

\section{DEMO DESCRIPTION}

In this demo, we present a model electric car performing automated driving and charging controlled by a central backend server. Adhering closely to the actual V-Charge system, the server is responsible for global scheduling of resources,

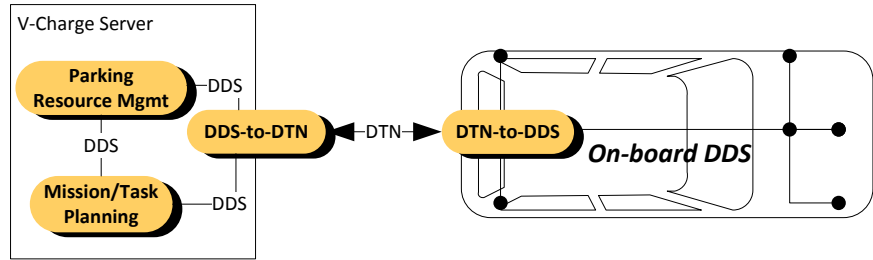

Figure 3. Communication Architecture in the V-Charge system

sending high-level commands, while the vehicle performs all other tasks automatically. The V2I communication over a WLAN link is based on the DDS middleware, resembling the real project. The car automatically maneuvers to the assigned destination, using the described line following and marker detection. The server application is constantly provided with status updates of the vehicle's position.

\section{Further Use CASES}

Although the primary use case of the mobile platform is as a demonstrator for the automated parking and charging proposed by the V-Charge project, it can also be seen as a versatile research and education platform for embedded and vehicular computing. The SBC, for instance, is able to run the OpenCV framework which can be used for several tasks like navigation. Additionally, different communication modules can be attached to the SBC, for example IEEE 802.15.4 radios as in a previously demonstrated disaster-recovery scenario [2]. The charging mechanism allows even long running automated experiments without requiring user interaction for changing batteries.

\section{CONCLUSION}

We proposed and demonstrated an automated parking and charging application for a model electric vehicle. Apart from the vehicle functionality, logistics and infrastructure support by a back-end responsible for global scheduling of resources has been integrated. For mission-control and monitoring purposes, V2I communication is deployed.

\section{ACKNOWLEDGMENT}

This project has received funding from the European Union's Seventh Framework Programme (FP7/2007-2013) under Grant Agreement Number [269916, V-Charge]. Further, we would like to thank Keno Garlichs, Alexander Willecke and Jan Käberich for their support.

\section{REFERENCES}

[1] P. Furgale et al. "Toward Automated Driving in Cities using Close-to-Market Sensors: An Overview of the VCharge Project." In: Proceedings of the IEEE Intelligent Vehicle Symposium (IV'13). Gold Coast, Australia: IEEE, June 2013, pp. 809-816.

[2] S. Rottmann et al. "Demo: Using DTN for Controlling A Vehicle With A Self Deployed WSN." In: Proceedings of the 5th Extreme Conference of Communication (ExtremeCom 2013). Thorsmork, Iceland, Aug. 2013. 